\title{
Effects of COVID-19 Outbreak in Image-Guided Biopsies in Brazil: An Epidemiological Study over 13 Years and 2 Million Biopsies
}

\author{
Marcela Juliano Silva Cunha ${ }^{1}$ (D) Marcelo Fiorelli Alexandrino da Silva ${ }^{1}$ • \\ Kaue Polizel Souza ${ }^{1}$ - Breno Boueri Affonso ${ }^{1}$ - Francisco Leonardo Galastri ${ }^{1}$ • \\ Felipe Nasser $^{1} \cdot$ Leonardo Guedes Moreira Valle $^{1} \cdot$ Rodrigo Gobbo Garcia $^{2}$. \\ Nelson Wolosker ${ }^{1}$
}

Received: 20 November 2021 / Accepted: 15 January 2022/Published online: 2 February 2022

(C) Springer Science+Business Media, LLC, part of Springer Nature and the Cardiovascular and Interventional Radiological Society of Europe (CIRSE) 2022

Dear Editor,

The Brazilian public health care system (SUS) provides complete coverage across the country. Nevertheless, during the COVID-19 pandemic, as the disease's epicenter, Brazil faced an unprecedented number of sudden hospital admissions, shortage of resources, and health workers' burn out [1]. Thus, according to governmental decisions, most public institutions had their non-urgent procedures and surgeries suspended during the outbreak waves to save economic and human resources to COVID-19 critically ill patients. In this scenario and similarly to other countries $[2,3]$, it was already expected that cancer diagnosis and treatment would be undermined. This study assesses Brazilian trends in image-guided biopsies in the SUS in the last 13 years and estimates how many potential missed diagnoses happened due to the pandemic in 2020 .

This retrospective study obtained the number of diagnostic procedures performed by interventional radiologists from the SUS Information system (DATASUS). This

All authors contributed to the study conception and design. Material preparation, data collection and analysis were performed by Marcelo Fiorelli Alexandrino da Silva, Kaue Polizel Souza and Breno Boueri Affonso. The first draft of the manuscript was written by Marcela Juliano Silva Cunha and Leonardo Guedes Moreira Valle and all authors commented on previous versions of the manuscript. All authors read and approved the final manuscript.

Marcela Juliano Silva Cunha

marcelajuliano90@gmail.com

1 Vascular Surgery Department at Hospital Israelita Albert Einstein, Rua Vapabussu 66 ap 402 Jd Aeroporto CEP, São Paulo, SP 04632-010, Brazil

2 Interventional Radiology Department at Hospital Israelita Albert Einstein, São Paulo, SP, Brazil public database includes information from all public health hospitals throughout the country, guaranteeing health support to about 170 million Brazilians.

As the DATASUS database uses secondary data, ethical approval and informed consent were not required according to resolution 510 of the Brazilian National Health Council.

ARIMA models $(1,1,0)$, which is a first-order autoregressive model with one order of nonseasonal differencing and a constant term, were adjusted for each series of biopsies evaluated, and the adjusted model was used to estimate the number of biopsies for 2020. Furthermore, the results are illustrated with plots of the series with the observed value for 2020 and the value expected by the adjusted models.

Based on the initial 12 years, it was estimated that there should have been 242,705 biopsies in 2020 (liver, prostate, kidney, thyroid, and lung) in SUS; however, the real number of biopsies that were performed was 160,238 (Fig. 1A), which is $66 \%$ of the expected number. Except for liver and kidney biopsies, which were decreasing over these years, the other ones consistently increased compared to 2008 (Fig. 1B-F). We believe that this discrepancy is due to the progressive improvement of imaging diagnosis of liver and kidney cancer, making biopsies necessary only for specific cases where this diagnosis is not possible by CT scan alone. Over time, CT scans have improved in technical quality and number in Brazil. Figure 2 shows the expenditure per year in U\$ dollars, referring exclusively to the costs of biopsies. The amount of money reimbursed for these procedures in 2020 was U\$2,365,449, the same value as ten years before.

Over 80,000 patients were no longer submitted to biopsies in the SUS in 2020. We can assume that the 

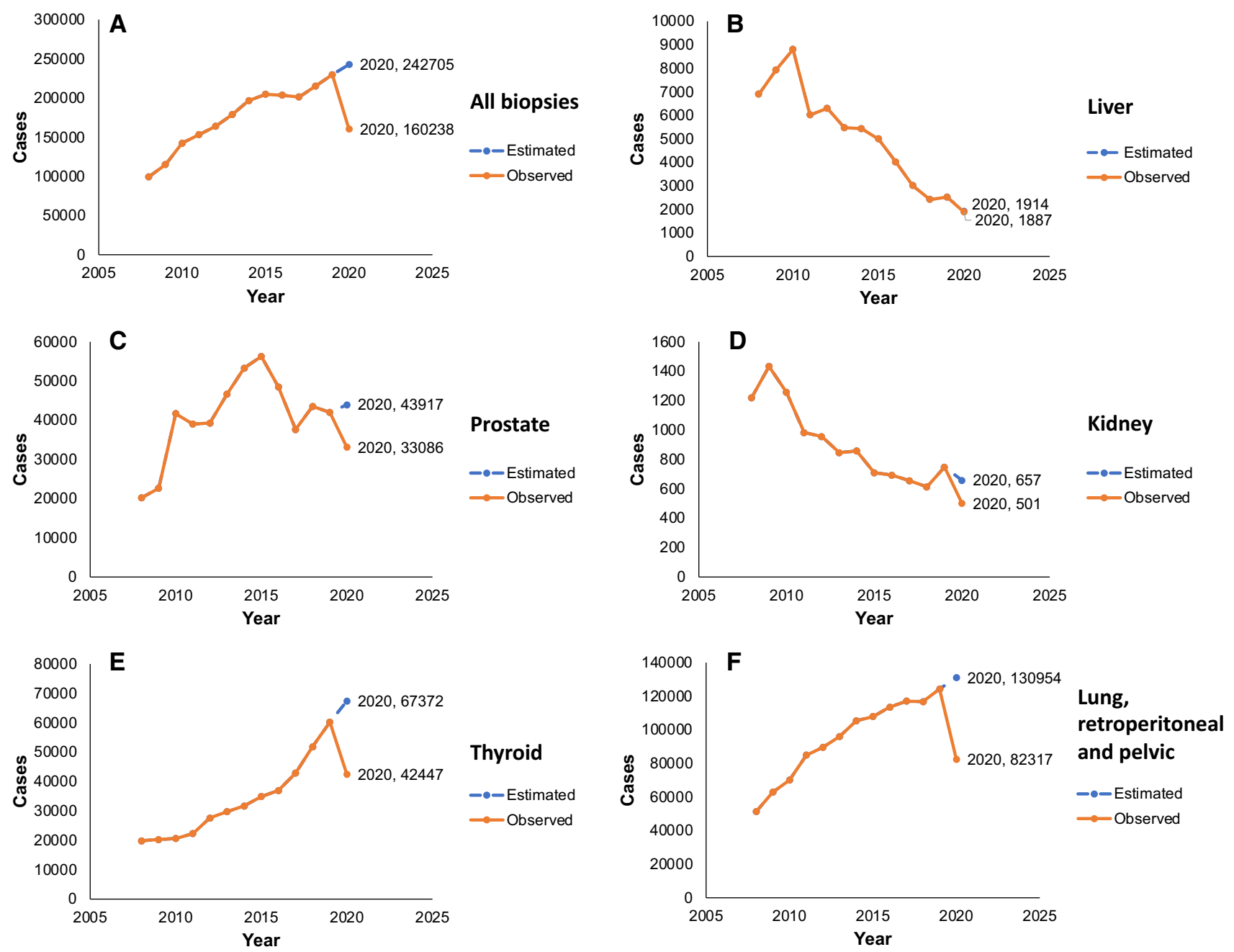

Fig. 1 Number of patients that were biopsied from 2008 to 2020 and the number that was expected for 2020, the COVID-19 first year

Fig. 2 Biopsies costs in U\$ dollars per year
Amout spent in U\$ dollars per year

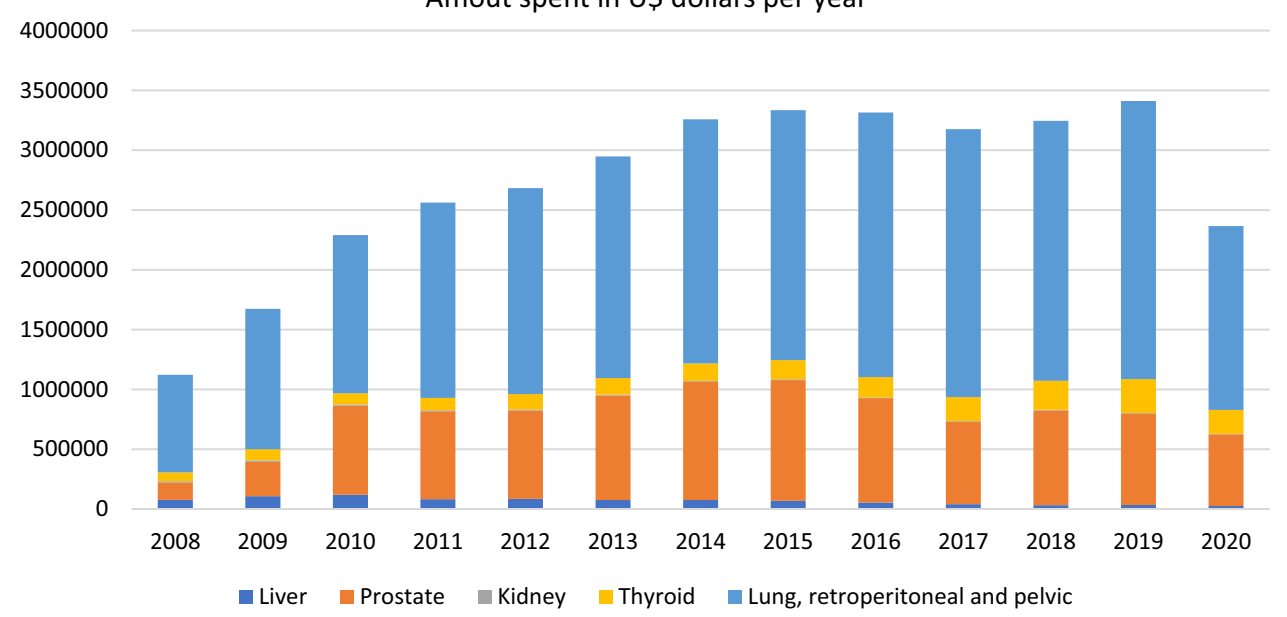


decrease in these screening tests corresponds to a decrease in cancer diagnoses and treatment. Based on this data, we expect an overload of oncological patients requiring care when the COVID-19 spread is controlled. As if that were not enough, it is expected that Brazil faces exacerbation of inequities due to the growing unemployment rates and that the public system turns out to be overburdened.

In the UK, cancer diagnoses are predicted to be $20 \%$ higher next year, leading to 6000 deaths [4]. This collateral loss could be even worse in a developing country like Brazil. Understanding these consequences of the COVID19 outbreak is imperative, and other countries' experiences should be role models for this damage control situation [5]. This will require a national planning effort to ensure that these future undiagnosed cancer cases will not be underestimated and mismanaged. Prompt government response would be needed to reduce distraction effects, avoiding even more unfavorable outcomes. Possible solutions would be to create campaigns and joint efforts, in the period between COVID-19 waves, to speed up the performance of these biopsies, reducing the bureaucratic process. Even during transmission peaks, diagnostic centers could have a specific location and days dedicated to perform these biopsies more safely. Besides, requests for this tests could be made during telehealth appointments, reducing patients unnecessary travel and exposition. Figure 2 shows that in 2020 , there was a considerable drop in the total amount spent on biopsies in SUS compared to previous years. This inconsistency raises concerns, emphasizing the possible burden expected for the following year in the public system.

Our study must be interpreted in the context of the study design. There are limitations related to the cross-sectional design and the possibility of inaccurate registries of DATASUS. However, the same biases might have played a role in previous years.

\section{Declarations}

Conflict of interest No conflicts of interest.

Informed consent The Institutional Review Board approved the request to waive the documentation of informed consent.

\section{References}

1. The Lancet. COVID-19 in Brazil: "So what?" Lancet. 2020; 395(10235):1461. https://doi.org/10.1016/S0140-6736(20)31095-3

2. Gurney JK, Millar E, Dunn A, et al. The impact of the COVID-19 pandemic on cancer diagnosis and service access in New Zealanda country pursuing COVID-19 elimination. Lancet Reg Heal-West Pacific. 2021;10: 100127. https://doi.org/10.1016/j.lanwpc.2021. 100127.

3. Bakouny Z, Paciotti M, Schmidt AL, Lipsitz SR, Choueiri TK, Trinh Q-D. Cancer screening tests and cancer diagnoses during the COVID-19 pandemic. JAMA Oncol. 2021;7(3):458. https://doi. org/10.1001/jamaoncol.2020.7600.

4. Lai A, Pasea L, Banerjee A, et al. Estimating excess mortality in people with cancer and multimorbidity in the COVID-19 emergency. BMJ Open. 2020. https://doi.org/10.1101/2020.05.27. 20083287.

5. Abdihamid O, Cai C, Kapesa L, Zeng S. The landscape of COVID19 in cancer patients: prevalence, impacts, and recommendations. Cancer Manag Res. 2020;12:8923-33. https://doi.org/10.2147/ CMAR.S272008.

Publisher's Note Springer Nature remains neutral with regard to jurisdictional claims in published maps and institutional affiliations. 